\title{
Ethnobotanical Uses of Non-cultivated Edible Fruit Species in the Department of Oussouye (South Senegal)
}

\author{
Yves Djihounouck $^{1}$, Doudou Diop ${ }^{2}$, César Bassene ${ }^{3}$, Seyni Sane ${ }^{1} \&$ Kandioura Noba $^{1}$ \\ ${ }^{1}$ Botany and Biodiversity Laboratory, Department of Plant Biology, Cheikh Anta Diop University of Dakar, BP \\ 5005, Senegal \\ ${ }^{2}$ Botany Laboratory, Institut fondamentale d'Afrique Noire (IFAN), Cheikh Anta Diop University of Dakar, BP \\ 206, Senegal \\ ${ }^{3}$ Section of Plant Production and Agronomy, Faculty of Agronomic Sciences, Aquaculture and Food \\ Technologies, Gaston Berger University of Saint Louis, BP 234, Senegal
}

Correspondence: Yves Djihounouck, Botany and Biodiversity Laboratory, Department of Plant Biology, Cheikh Anta Diop University of Dakar, BP 5005, Senegal. Tel: 221-772-096-605. E-mail: djihounouck01 @ yahoo.fr

Received: April 30, 2021

doi:10.5539/jfr.v10n4p16
Accepted: June 23, $2021 \quad$ Online Published: July 17, 2021

URL: https://doi.org/10.5539/jfr.v10n4p16

\begin{abstract}
Forests are an immense reservoir of biological resources and provide the local population with subsistence needs, especially for edible fruits. This study contributes to a better knowledge of the use modes of non-cultivate edible plant species in the area of Kasa, traditional name for the department of Oussouye. Ethnobotanical surveys, based on an interview guide, oral discussions and direct observations were conducted among 178 people from the department of Oussouye, stronghold of the Diola ethnic group. A factorial correspondence analysis highlighted the relationship between species and categories of use. The frequency of citation, informant consensus factor and use value showed the socio-cultural importance of the species. The data collected identified 62 edible species divided into 31 families and 54 genera. The fruit species inventoried are used for different purposes. They are a food source with $62 \%$ of citations, energetic $19 \%$, technological $14 \%$, medicinal $13 \%$, cultural $6 \%$ and agronomic $2 \%$ for the populations. Two species stand out for their high use value factor (UVt). These were Elaeis guineensis (12.24) and Borassus aethiopum (7.56). In addition to their use value, species such as Mangifera indica, Neocarya macrophylla, Parkia biglobosa, Anacardium occidentale, Ceiba pentandra, Parinari excelsa, stood out for their categories and organs used. These results inform us about the level of use of fruit species for different needs and open up avenues for research in sustainable management of this resource with the aim of reducing poverty.
\end{abstract}

Keywords: plant species, Diola, added value, potential uses, Kasa

\section{Introduction}

Tropical forests constitute an immense reservoir of biological resources for pharmacopoeia, food, construction, timber industry and handicrafts among local populations. Thus, they provide local populations with subsistence needs. In rural areas, people's lives depend on non-timber and timber forest products (Bikoué \& Essomba, 2007).

For a long time, the place occupied by non-timber forest products (NTFPs) and timber products in development policies has been minimal and often limited to indigenous uses. However, the contribution of these products to food security and primary health care has been amply demonstrated, as nearly $80 \%$ of the population in developing countries use them for health care or food (Allabi et al., 2011). In this sense, traditional medicine relieves more than $70 \%$ of the populations of the Third World (Malaisse, 1992) and 80\% of the African peoples (Jiofack et al. , 2009). Thus, traditional medicine has become part of the culture of African populations although it still remains informal (Sofowara, 1982). In Senegal, 550 plants are considered medicinal and toxic in the pharmacopoeia (Kerharo \& Adam, 1974). However, the use of plants requires a wider and deeper knowledge in order to be able to integrate them into the socio-economic development processes of the populations.

In Africa, non-cultivate plants are used daily by the great majority of peasant peoples (Grivetti et al., 1987). These plants essentially contribute to the food and survival of rural people by providing scarce nutrients in their diet (Ayessou et al., 2011). In Senegal, the species most used by the populations have been determined by 
ethnobotanical methods based on inventories and uses of species in domains such as food and medicine (Diop, 2011; Dieng, 2017; Djihounouck et al, 2018). However, this assessment does not cover the department of Oussouye even though fragmentary data on the uses of a limited number of species in this locality happened to be collected. Data collection on the uses of plant species is an important step in supporting decentralized programs for the conservation and development of forest resources (Ayessou et al., 2009; 2011; Diop et al., 2010), hence the need to conduct this study among the populations in the department of Oussouye in order to contribute to better management of these natural resources. This study contributes to the knowledge of the uses of edible fruit species in the Kasa area. To this end, the specific objectives of the study are to:

$\sim$ identify the different categories of uses of the inventoried wild edible species;

$\sim$ describe their uses to optimize the contribution of forest genetic resources to sustainable socio-economic development;

\section{Methodology}

The department of Oussouye is located in southwestern Senegal and covers $891 \mathrm{~km}^{2}$ i. e. $12.14 \%$ of the area of the Ziguinchor region. It is bordered to the east by the Kamobeul marigot, the north by the Casamance River, the west by the Atlantic Ocean and Cape Roxo, and the south by Guinea Bissau. It includes five communes that total seventy-five (75) villages, with a population of 48.331 inhabitants in 2013 (ANSD, 2015). (Figure 1)

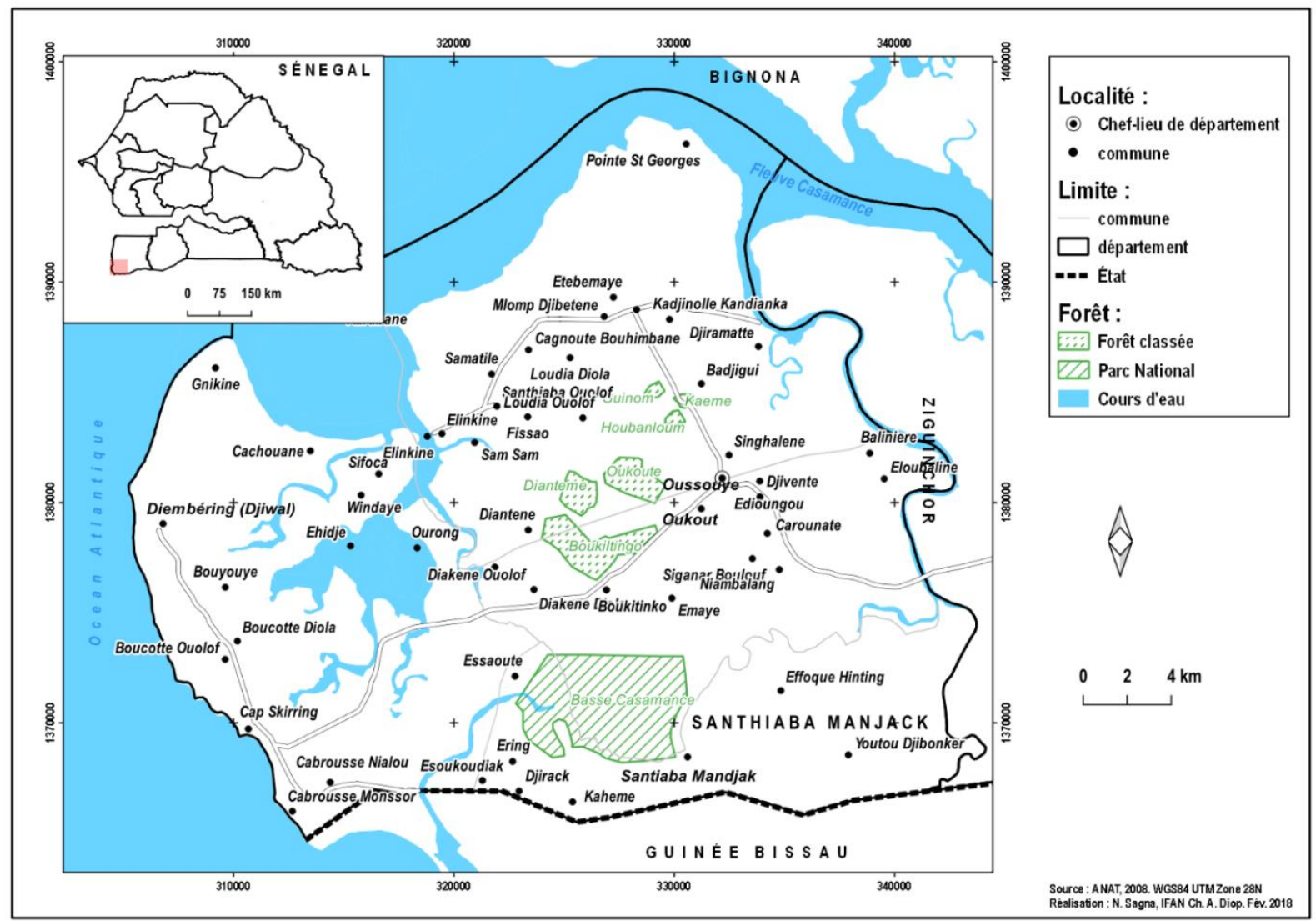

Figure 1. Map of the administrative division, hydrographic network and forest areas of the Oussouye department (Source: ANAT, 2008. WGS84 UTM Zone 28N. Production: N. Sagna Ifan Ch. A. Diop Feb. 2018).

The study population is made up of the Diola who are the majority ethnic group in the department of Oussouye. The language spoken by this ethnic group bears the same name (Diola) and is made up of several dialects. When the dialect is not mastered during the ethnobotanical survey, we call in an interpreter to ensure good communication. A first interview was conducted with randomly selected people in villages. This contact visit allowed us to get an idea of how the species studied contribute to the life of the population and select the areas of activity. The choice of the villages where the surveys should be conducted was made taking into account the local forest resources that allow harvesting activities. The newly created villages called batogat were not chosen because they do not have cultural originality due to the loss of ancestral values. Villages affected by the 
Casamance conflict were excluded from the survey. Based on those criteria, 34 villages were selected for data collection. These villages are located in five (5) traditional communities (An Alufay, Esulalu, Ejamat, Dyiwat and Her or Haer). Each community is distinguished from the others in its dialect, organization and social practices. The choice of interviewees was non-probabilistic and their identification was done for convenience. Their selection by study area and village depended on their availability. They were chosen with the help of the village Chiefs, targeting individuals whose activity, experience or status was related to the topic. They were herders, gatherers, traditional family caterers, and farmers. The interviews were carried out between 2012 and 2014 mainly with indigenous adults, with a preference for men who have a better knowledge of non-cultivate species and who have experience in their mode of use. The choice of these people was made in order to reduce the risk of uncertain or vague answers. Data were collected through semi-structured interviews and casual conversations.

Semi-structured interviews were conducted using an interview guide which included the following headings: interviewee identification, form of use, socio-cultural importance of the species, and description of use modes. Casual conversations were used to estimate responses and gather new information (Martin, 1995).

The local name of each plant studied was transcribed into local language based on specialized documents (Adam, 1970; Berhaut, 1967) or by using the Diola alphabet codified with reference to the local language of the $A n$ Alufaye community.

The identification of fruit plants was done either on site or in the botany laboratory of IFAN and the botany and biodiversity laboratory of the Department of Plant Biology (BV) using the various flora of Senegal by Berhaut $(1979 ; 1976 ; 1975 a ; 1975 b ; 1974 ; 1971 ; 1967)$ and Van Der Berghen (1988), and the works of Hawthorne and Jongkind (2006), Arbonnier, (2002) and Hutchinson and Dalziel (1954), Wieschus (2000) and the herbarium collections of Dakar and IFAN. The nomenclature adopted is that of the database of the Conservatoire et Jardin Botanique (C.J.B) of the city of Geneva, which is regularly updated (Lebrun \& Stork, 1997; 1995; 1992; 1991).

Qualitative data were processed using the content analysis method (Faucault, 1966; Négura, 2006). This process consists of a systematic and methodical examination of textual and / or visual documents. Applied to qualitative data, it captures the contextual dimension of these documents and highlight the categorical determinants.

Quantitative data were processed according to descriptive statistics using response rate (Van den Eyden et al., 1994; Cotton, 1996).

The data obtained were analyzed with Epi Info software and the results were processed by Excel spreadsheet which allowed us to establish cross-tabulations [species /organs], [species/use], [species/method of collection], [species/stage of development] and the R software (Core Team, 2017) with the factoextra package (Version: 1.0.5), for Factorial Correspondence Analysis (FCA) allowing to study the relationships between different species and categories of use. A test of independence of Chi2 with a threshold of significance of $5 \%$ was previously carried out in order to verify the independence between the different species and the categories of use. The information collected was analyzed on the basis of ethnobotanical indicators.

$>$ Frequency of Citation (FC) is used to determine the level of use of different species in a category and ranges from 0 to 100 . The value 0 indicates that the species is not used in this category and 100 when the species is used in this category by all interviewees. It is expressed by the following formula:

$\mathrm{FC}=(\mathrm{S} / \mathrm{N}) \mathbf{1 0 0 \%}$ where:

- $\mathbf{S}$ : number of citations of a species in a category;

- $\mathbf{N}$ : total number of informants.

$>$ Informant Consensus Factor (ICF) measures the variability of species use forms (Trotter \& Logan, 1986). Always between 0 and 1, the value of this ICF is high when only one or a small number of species are cited by a large proportion of informants for a specific use category. Conversely, the greater the diversity of species cited for the same use, the closer the value will be to 0 . The ICF is calculated from the following formula:

ICF $=$ Nur - Nt $/($ Nur - 1) where,

- Nur (user-reports number) is the number of uses indicated in a given category;

- Nt (number of taxa) the number of species involved in this same use category.

$>$ Use Value $\left(\mathbf{U V}_{(\mathbf{K})}\right)$ is a way of expressing the importance of each family or species for the population intervieweed. It significantly identifies the species with a high use value in a given environment (Dossou et al ., 
2012). It was calculated according to the method used by Philips \& Gentry (1993) and Camou-Guerrero and his team (2008) using the following formula:

$$
\mathbf{U V}_{(\mathbf{K})}=\frac{\sum_{\mathbf{i}}^{\mathrm{n}} \mathbf{s i}}{\mathbf{n}} \text { where, }
$$

- $\mathbf{U V} \mathbf{V}_{(\mathbf{k})}$ is the ethnobotanical use value of species k within a given use category,

- $\mathbf{s i}$ is the number of uses assigned by respondent $\mathrm{i}$ within this category,

- $\mathbf{n}$ is the number of respondents for a given category of use.

The total use value $\left(\mathbf{U V}_{\mathbf{t}}\right)$ of species $\mathrm{k}$ is then calculated by summing the use values of that species within the different use categories by the formula:

$$
\mathbf{U V}_{\mathbf{t}}=\sum_{\mathbf{1}}^{\mathbf{p}} \mathbf{U V} \text { where, }
$$

- $\mathbf{U V} \mathbf{V}_{\mathbf{t}}$ represents the total use value of the species,

- UV is the use value of the species for a given use category, $\mathrm{p}$ is the number of use categories.

\section{Results}

Thirty-four (34) villages were visited as part of this survey. The number of interviewees varied from 38 to 4 per area and 2 to 6 in each village. In total, eighty-two (82) informants were interviewed individually or in groups. This amounts to identifying one hundred and seventy-eight (178) interviewees in the five traditional areas.

\subsection{Use Values}

Data collected made it possible to inventory 62 non-cultivate edible fruit plant species distributed in 31 families. Apocynaceae and Annonaceae are the most represented with 6 species each. They are followed by Anacardiaceae (5 species) and Chrysobalanaceae, Arecaceae and Rubiaceae (4 species each). The other families are made up of Sapindaceae, Caesalpiniaceae, Moraceae, Celastraceae (3 species each), Bombacaceae and Zingiberaceae ( 2 species). The remaining 19 families are each represented by 1 species. (Table 1)

Generic diversity is also important with 54 genera in total. The family Anacardiaceae is the most diverse with 5 genera, followed by Annonaceae, Arecaceae and Rubiaceae with 4 genera each, Apocynaceae, Chrysobalanaceae, Sapindaceae, Celastraceae, Caesalpiniaceae with 3 genera and Moraceae, Bombacaceae with 2 genera each. The remaining families are represented by only one genus each. (Table 1)

These species are grouped in 6 use categories which are food, medicinal, technological, agronomic, energetic and cultural. The organs used are 7 in total, namely fruit, wood, leaf, root, bark, exudate and flower. (Table 1)

The analysis of the results shows:

- 6 use categories concerning 3 species: Elaeis guineensis, Neocarya macrophylla and Parinari excelsa;

- 5 use categories represented by 8 species;

- 4 use categories for 17 species;

- 3 use categories for 9 species;

- 2 use categories for 15 species;

- $\quad$ and 1 use category for 10 species.

Regarding the organs harvested, the results indicate:

- 6 organs used in Elaeis guineensis, Mangifera indica and Cola cordifolia ;

- 5 organs exploited in 15 species;

- 4 organs in 15 species;

- 3 organs in 6 species;

- 2 organs in 13 species;

- and 1 organ in 10 species.

The use values are:

- $\quad$ high in two palm trees: Elaeis guineensis with 12.24 and Borassus aethiopum with 7.56; 
- medium [3.87-2.35] in Neocarya macrophylla , Mangifera indica , Parkia biglobosa, anacardium occidentale , Ceiba pentandra , Parinari excelsa, Dialium guineense and Adansonia digitata .

- low to very low for the rest of the species.

These species are useful to the population in several fields: food, medicine, technology, agronomy, energy and culture. The organs used are fruits, wood, leaves, bark, roots, flowers and exudate.

The food use of the species is the most frequent, followed by the medicinal use. Our results show that there is not a close proportionality between the use value of the species and its organs used. A species can be highly prized for one organ in a given environment, while another species with multiple uses is poorly known (Table 1). For example, Borassus aethiopum (UVt $=7.56$ ) with five use categories and four organs used, is highly used while Parinari excelsa $(\mathrm{UVt}=2.59)$ with six use categories and five organs exploited, is very little sought after.

Table 1. Categories, organs used and use value of species

\begin{tabular}{|c|c|c|c|c|c|}
\hline Families & Scientific name & Local Name & Use categories & $\begin{array}{l}\text { Organs } \\
\text { used }\end{array}$ & $\begin{array}{l}\text { Use } \\
\text { value } \\
\left(\mathbf{U V}_{\mathrm{t}}\right)\end{array}$ \\
\hline Arecaceae & Elaeis guineensis Jacq. & ka bekel, ka hiit & $\begin{array}{l}\text { Fo, Med, Tech, } \\
\text { Ag, En, Cul }\end{array}$ & $\begin{array}{l}\text { Fr, Wo, Le, } \\
\text { Ro, Ex, Fl }\end{array}$ & 12.24 \\
\hline Arecaceae & Borassus aethiopum (L.) Mart. & ka lahaay & $\begin{array}{l}\text { Al, Med, Tech, } \\
\text { En, Cul }\end{array}$ & $\begin{array}{l}\text { Fr, Wo, } \\
\text { Le, Ro, Fl }\end{array}$ & 7.56 \\
\hline $\begin{array}{l}\text { Chrysobal } \\
\text { anaceae }\end{array}$ & $\begin{array}{l}\text { Neocarya macrophylla (Sabine) } \\
\text { Prance }\end{array}$ & $\begin{array}{l}\text { bu bita, bu nafay, } \\
\text { beel }\end{array}$ & $\begin{array}{l}\text { Fo, Med, Tech, } \\
\text { Ag, En, Cul }\end{array}$ & $\begin{array}{l}\text { Fr, Wo, } \\
\text { Le, Ba, Ro }\end{array}$ & 3.87 \\
\hline $\begin{array}{l}\text { Anacardia } \\
\text { ceae }\end{array}$ & Mangifera indica L. "Perse" & $\begin{array}{l}\text { bu mangali bu } \\
\text { jóoluay, } \\
\text { bu mangu bu } \\
\text { jóoluay }\end{array}$ & $\begin{array}{l}\text { Fo, Med, Tech, } \\
\text { Ag, En }\end{array}$ & $\begin{array}{l}\text { Fr, Wo, } \\
\text { Le, Ba, Ro, Fl }\end{array}$ & 3.76 \\
\hline $\begin{array}{l}\text { Mimosacea } \\
e\end{array}$ & Parkia biglobosa (Jaq.) Benth. & bu nalay, bu níók & $\begin{array}{l}\text { Fo, Med, Tech, } \\
\text { Ag, En }\end{array}$ & $\begin{array}{l}\text { Fr, Wo, } \\
\text { Le, Ba, Ro }\end{array}$ & 3.7 \\
\hline $\begin{array}{l}\text { Anacardia } \\
\text { ceae }\end{array}$ & Anacardium occidentale $\mathrm{L}$. & bu talakasa, bu bisa & $\begin{array}{l}\text { Fo, Med, Tech, } \\
\text { Ag, En }\end{array}$ & $\begin{array}{l}\text { Fr, Wo, } \\
\text { Le, Ba, Ro }\end{array}$ & 3.5 \\
\hline $\begin{array}{l}\text { Bombacac } \\
\text { eae }\end{array}$ & Ceiba pentandra (L.) Gaertn & bu sana & $\begin{array}{l}\text { Fo, Med, Tech, } \\
\text { Ag, En }\end{array}$ & $\begin{array}{l}\text { Fr, Wo, } \\
\text { Le, Ba, Ro }\end{array}$ & 3.23 \\
\hline $\begin{array}{l}\text { Chrysobal } \\
\text { anaceae }\end{array}$ & Parinari excelsa Sabine & $\begin{array}{l}\text { bu wel, bu fujay, e } \\
\text { liik }\end{array}$ & $\begin{array}{l}\text { Fo, Med, Tech, } \\
\mathrm{Ag}, \text { En, Cul }\end{array}$ & $\begin{array}{l}\text { Fr, Wo, Le, } \\
\text { Ba, Ro }\end{array}$ & 2.59 \\
\hline $\begin{array}{l}\text { Caesalpini } \\
\text { aceae }\end{array}$ & Dialium guineense Willd. & bu fulan, bu foyitay & $\begin{array}{l}\text { Fo, Med, Tech, } \\
\text { En }\end{array}$ & $\begin{array}{l}\text { Fr, Wo, Le, } \\
\text { Ro }\end{array}$ & 2.43 \\
\hline $\begin{array}{l}\text { Bombacac } \\
\text { eae }\end{array}$ & Adansonia digitata $\mathrm{L}$. & bu kona, bu baak & $\begin{array}{l}\text { Fo, Med, Tech, } \\
\text { Cul }\end{array}$ & Fr, Le, Ba & 2.35 \\
\hline $\begin{array}{l}\text { Nympheac } \\
\text { eae }\end{array}$ & Nymphaea spp & bu kikif, e bahál & Fo, Tech & Fr, Le, Ro & 1.99 \\
\hline $\begin{array}{l}\text { Apocynace } \\
\text { ae }\end{array}$ & $\begin{array}{l}\text { Landolphia dulcis (Sabine) } \\
\text { Pichon }\end{array}$ & $\begin{array}{l}\text { bu bot, bu ñohol, bu } \\
\text { ñohon }\end{array}$ & Fo, Med, Tech & $\begin{array}{l}\text { Fr, Wo, Le, } \\
\text { Ba, Ro }\end{array}$ & 1.98 \\
\hline $\begin{array}{l}\text { Annonacea } \\
e\end{array}$ & $\begin{array}{l}\text { Xylopia aethiopica (Dunal) A. } \\
\text { Rich. }\end{array}$ & bu lew ba finoe & $\begin{array}{l}\text { Fo, Med, Tech, } \\
\text { En }\end{array}$ & $\begin{array}{l}\text { Fr, Wo, Le, } \\
\text { Ro }\end{array}$ & 1.85 \\
\hline $\begin{array}{l}\text { Annonacea } \\
e\end{array}$ & Uvaria chamae P. Beauv. & bu lew & $\begin{array}{l}\text { Fo, Med, Tech, } \\
\text { En }\end{array}$ & $\begin{array}{l}\text { Fr, Wo, Le, } \\
\text { Ba, Ro }\end{array}$ & 1.74 \\
\hline $\begin{array}{l}\text { Sapindace } \\
\text { ae }\end{array}$ & $\begin{array}{l}\text { Aphania senegalensis (Juss. Ex } \\
\text { Poir.) Radlk. }\end{array}$ & bu ul & $\begin{array}{l}\text { Fo, Med, Tech, } \\
\text { En }\end{array}$ & $\begin{array}{l}\text { Fr, Wo, Le, } \\
\text { Ro }\end{array}$ & 1.74 \\
\hline $\begin{array}{l}\text { Sapindace } \\
\text { ae }\end{array}$ & Allophyllus africanus P. Beauv. & $\begin{array}{l}\text { bu singilit, bu hul a } \\
\text { mata, bu fankahen }\end{array}$ & $\begin{array}{l}\text { Fo, Med, Tech, } \\
\text { En }\end{array}$ & $\begin{array}{l}\text { Fr, Wo, Le, } \\
\text { Ro }\end{array}$ & 1.66 \\
\hline $\begin{array}{l}\text { Apocynace } \\
\text { ae }\end{array}$ & $\begin{array}{l}\text { Saba senegalensis (A. DC.) } \\
\text { Pichon }\end{array}$ & bu híndik, bundok & $\begin{array}{l}\text { Fo, Med, Tech, } \\
\text { En }\end{array}$ & $\begin{array}{l}\text { Fr, Wo, Le, } \\
\text { Ex }\end{array}$ & 1.63 \\
\hline Arecaceae & Phoenix reclinata Jacq. & $\begin{array}{l}\text { bu faba faba, bu } \\
\text { sanjab, } \\
\text { bu juka, bu jak }\end{array}$ & $\begin{array}{l}\text { Al, Med, Tech, } \\
\text { Ass }\end{array}$ & $\begin{array}{l}\text { Fr, Wo, Le, } \\
\text { Ro }\end{array}$ & 1.62 \\
\hline
\end{tabular}




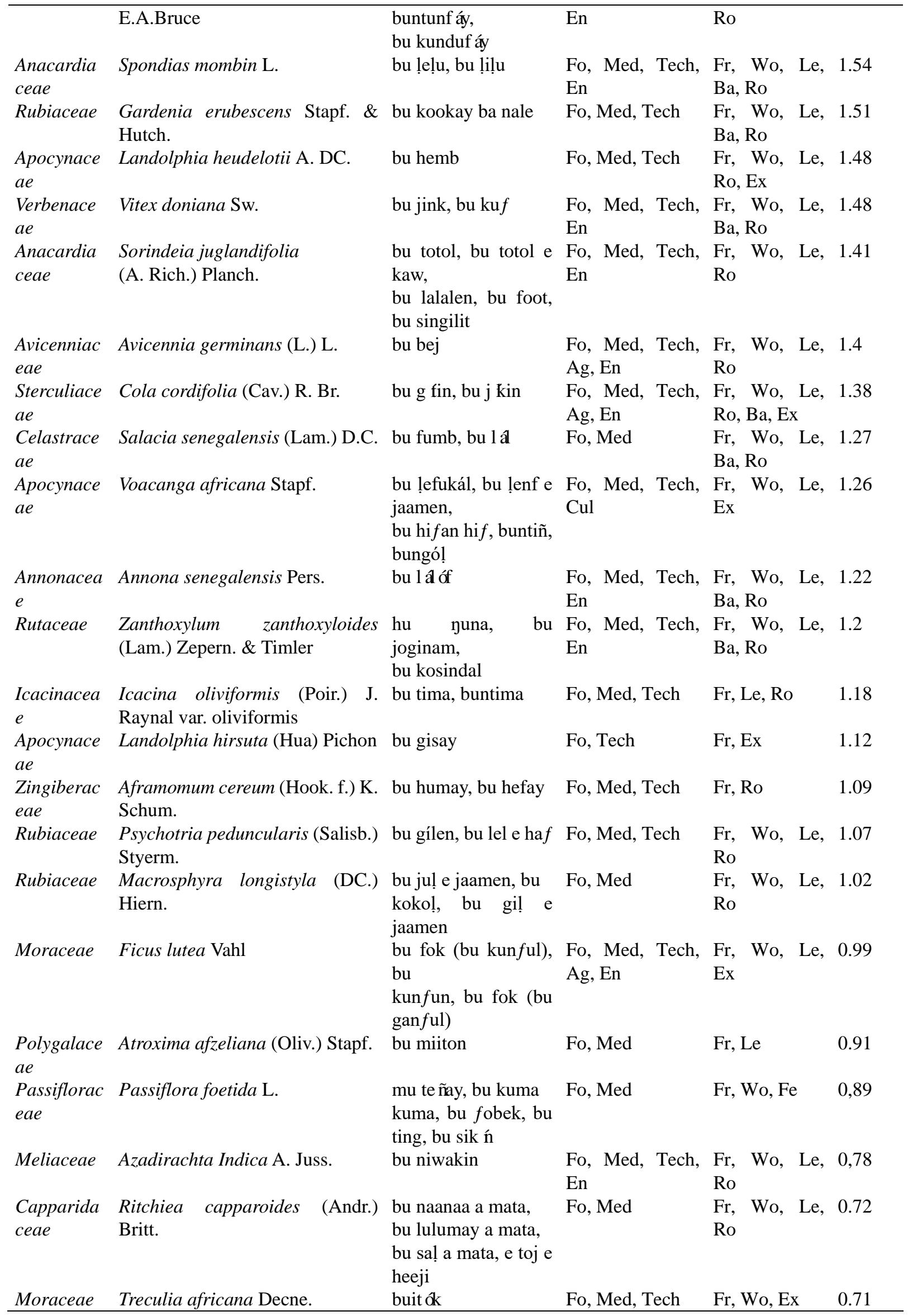




\begin{tabular}{|c|c|c|c|c|c|}
\hline $\begin{array}{l}\text { Chrysobal } \\
\text { anaceae }\end{array}$ & $\begin{array}{l}\text { Chrysobalanus ellipticus Sol. ex } \\
\text { Sabine }\end{array}$ & $\begin{array}{l}\text { bu noñ, bu sima, } \\
\text { bu u! e jakal }\end{array}$ & $\begin{array}{l}\text { Fo, Med, Tech, } \\
\text { En }\end{array}$ & Fr, Wo & 0.61 \\
\hline $\begin{array}{l}\text { Cucurbitac } \\
\text { eae }\end{array}$ & $\begin{array}{l}\text { Cucumis metuliferus E. Mey. ex } \\
\text { Naudin }\end{array}$ & $\begin{array}{l}\text { bu konkombra } \\
\text { firika, } \\
\text { bu konkombura, e } \\
\text { gil e jaamen }\end{array}$ & Fo, Med & Fr, Le & 0.61 \\
\hline Ebenaceae & Diospyros ferrea (Willd.) Bakh. & $\begin{array}{l}\text { bu wing a } \\
\text { ligen, lalalen }\end{array}$ & Fo, Med, En & Fr, Wo & 0.35 \\
\hline Clusiaceae & Mammea africana Sabine & báál & Fo, Med & Fr, Le & 0.33 \\
\hline $\begin{array}{l}\text { Caesalpini } \\
\text { aceae }\end{array}$ & Detarium senegalense J.F. Gmel. & $\begin{array}{l}\text { bu gawuj, bu } \\
\text { bunkut ba teñey }\end{array}$ & Fo, Med & Fr, Le & 0.29 \\
\hline $\begin{array}{l}\text { Sapindace } \\
\text { ae }\end{array}$ & Pancovia bijuga Willd. & buntañ, bu luñay & Fo, Med, En & Fr, Le, Wo & 0.28 \\
\hline $\begin{array}{l}\text { Caesalpini } \\
\text { aceae }\end{array}$ & Tamarindus indica $\mathrm{L}$. & bu dahar & Fo, Med & Fr, Le & 0.22 \\
\hline $\begin{array}{l}\text { Apocynace } \\
\text { ae }\end{array}$ & Landolphia owariensis P. Beauv. & bu tiyok, bu tik & Fo, Med & Fr, Ro & 0.17 \\
\hline $\begin{array}{l}\text { Zingiberac } \\
\text { eae }\end{array}$ & $\begin{array}{l}\text { Aframomum elliotii (Bak.) K. } \\
\text { Schum. }\end{array}$ & $\begin{array}{l}\text { bu humay e kobol, } \\
\text { bu hefay e kobol }\end{array}$ & Fo & $\mathrm{Fr}$ & 0.13 \\
\hline $\begin{array}{l}\text { Euphorbia } \\
\text { ceae }\end{array}$ & $\begin{array}{l}\text { Drypetes floribunda (Müll. Arg.) } \\
\text { Hutch. }\end{array}$ & bu huuta, bunkól & Fo & Fr & 0.12 \\
\hline Arecaceae & $\begin{array}{l}\text { Calamus deerratus G. Mann \& } \\
\text { H. Wendl. }\end{array}$ & ka hihá, ka ḷiyá & Fo, Tech & Fr, Wo & 0.12 \\
\hline $\begin{array}{l}\text { Annonacea } \\
e\end{array}$ & $\begin{array}{l}\text { Monanthotaxis barteri (Baill.) } \\
\text { Verdc. }\end{array}$ & bu lew ba yine & Fo & Fr & 0.11 \\
\hline Sapotaceae & $\begin{array}{l}\text { Synsepalum brevipes (Baker) T. } \\
\text { D. Penn. }\end{array}$ & bundukul & Fo & $\mathrm{Fr}$ & 0.09 \\
\hline $\begin{array}{l}\text { Rhamnace } \\
\text { ae }\end{array}$ & Ziziphus mauritiana Lam. & sidem & Fo & $\mathrm{Fr}$ & 0.09 \\
\hline $\begin{array}{l}\text { Annonacea } \\
e\end{array}$ & Uvaria thomasii Sprag. Et Hutch. & bu lew bu jal & Fo, Med & Fr, Ro & 0.09 \\
\hline $\begin{array}{l}\text { Annonacea } \\
e\end{array}$ & $\begin{array}{l}\text { Annona glauca Schumach. \& } \\
\text { Thonn. }\end{array}$ & bu lala & Fo, Med & Fr, Ro & 0.07 \\
\hline Ulmaceae & $\begin{array}{l}\text { Celtis toka (Forssk.) Hepper \& } \\
\text { J.R.I. Wood }\end{array}$ & buwintol & Fo & $\mathrm{Fr}$ & 0.06 \\
\hline Moraceae & Ficus capensis Thunb. & bu fok & Fo & $\mathrm{Fr}$ & 0.06 \\
\hline $\begin{array}{l}\text { Chrysobal } \\
\text { anaceae }\end{array}$ & $\begin{array}{l}\text { Chrysobalanus orbicularis } \\
\text { Schumach. }\end{array}$ & nu loña & Fo & $\mathrm{Fr}$ & 0.05 \\
\hline $\begin{array}{l}\text { Zygophylla } \\
\text { ceae }\end{array}$ & Balanites aegyptiaca (L.) Del. & & Fo & Fr & 0.02 \\
\hline $\begin{array}{l}\text { Anacardia } \\
\text { ceae }\end{array}$ & $\begin{array}{l}\text { Pseudospondias microcarpa (A. } \\
\text { Rich.) Engl. }\end{array}$ & bu nunu & Fo & $\mathrm{Fr}$ & 0.02 \\
\hline
\end{tabular}

Fo: food; Med: medicinal; Tech: technological; Ag: agronomic; En: energetic; $\mathbf{C u}$ : cultural.

$\mathbf{F r}$ : fruit; Le : leaf ; Ba : bark; Wo : wood ; Ro : root; Ex : exudate; Fl : flower.

\subsection{Use Categories}

Non-cultivate fruit species can be useful in several ways. The results on their use modes show 6 categories of use (Table 2). These are:

- food where all species are mentioned;

- medicinal with 52 useful species;

- technological with 39 species;

- $\quad$ energetic with 27 species;

- $\quad$ agronomic with 10 species; 
- cultural with 9 species.

The most used species in these different categories are: Elaeis guineensis with 378 citations, Borassus aethiopum with 343 citations, Mangifera indica with 266 citations, Parkia biglobosa with 227 citations, Ceiba pentandra with 226 citations, Neocarya macrophylla with 212 citations and Parinari excelsa with 210 citations (Table 2).

The frequency of citation (Table 2) is greater than $50 \%$ in:

- 38 species in the food sector;

- 9 species in the energy sector;

- 6 species in the technological sector;

- 4 species in the cultural sector;

- 1 species in the medicinal $f$ sector and less than $50 \%$ in agronomy.

This frequency of citation of species by populations can be high, medium or low depending on the category of use. For example, Borassus aethiopum, Phoenix reclinata are highly used in cultural field whereas they are not in medicine. A low use value may be due to a lack of knowledge about the plant or the disappearance of certain species due to forest erosion.

Table 2. Frequency of citation of the different use categories of non-cultivate edible fruit species

\begin{tabular}{|c|c|c|c|c|c|c|c|}
\hline \multirow[t]{2}{*}{ Scientific name } & \multirow{2}{*}{$\begin{array}{l}\text { Number of } \\
\text { citation }\end{array}$} & \multicolumn{6}{|c|}{ Frequency of Citation (FC) in \% } \\
\hline & & Fo & Med. & Tech & Ag & En & Cul \\
\hline Mangifera indica & 266 & 100 & 57 & 28 & 43 & 96 & \\
\hline Elaeis guineensis & 378 & 100 & 49 & 100 & $65 \%$ & 100 & 99 \\
\hline Landolphia dulcis & 127 & 100 & 49 & 6 & & & \\
\hline Parkia biglobosa & 227 & 100 & 44 & 24 & 18 & 90 & \\
\hline Nauclea latifolia & 126 & 100 & 39 & 4 & & $55 \%$ & \\
\hline Adansonia digitata & 163 & 100 & 38 & 57 & & & 3 \\
\hline Neocarya macrophylla & 221 & 100 & 38 & 16 & 5 & 96 & 4 \\
\hline Anacardium occidentale & 197 & 100 & 34 & 1 & 7 & 98 & \\
\hline Aphania senegalensis & 141 & 100 & 34 & 1 & & 37 & \\
\hline Uvaria chamae & 134 & 100 & 28 & 24 & & 55 & \\
\hline Saba senegalensis & 112 & 100 & 26 & 22 & & 1 & \\
\hline Salacia senegalensis & 101 & 100 & 23 & & & & \\
\hline Borassus aethiopum plant & 143 & 100 & 21 & 100 & & 98 & 100 \\
\hline Ceiba pentandra & 126 & 100 & 21 & 80 & 60 & 9 & 54 \\
\hline Landolphia heudelotii & 114 & 100 & 21 & 18 & & & \\
\hline Annona senegalensis & 97 & 100 & 15 & 4 & & & \\
\hline Parinari excelsa & 210 & 100 & 60 & 32 & 1 & 78 & 33 \\
\hline Vitex doniana $\mathrm{Sw}$ & 115 & 100 & 10 & 60 & & 18 & \\
\hline Aframomum cereum & 90 & 100 & 9 & 1 & & & \\
\hline Dialium guineense & 189 & 100 & 7 & 34 & & 89 & \\
\hline Nymphaea spp & 84 & 100 & 1 & 1 & & & \\
\hline Allophyllus africanus & 137 & 98 & 6 & 5 & & 59 & \\
\hline Phoenix reclinata & 134 & 96 & 1 & 5 & & & 61 \\
\hline Xylopia aethiopica & 133 & 94 & 44 & 21 & & 4 & \\
\hline Macrosphyra longistyla & 80 & 93 & 5 & & & & \\
\hline Sorindeia juglandifolia & 113 & 91 & 7 & 2 & & 37 & \\
\hline Atroxima afzeliana & 74 & 89 & 1 & & & & \\
\hline Mombin spondias & 121 & 88 & 4 & 52 & & 4 & \\
\hline Icacina oliviformis var. oliviformis & 93 & 87 & 23 & 4 & & & \\
\hline Cola cordifolia & 104 & 82 & 22 & 16 & 1 & 6 & \\
\hline Passiflora foetida & 70 & 80 & 5 & & & & \\
\hline Gardenia erubescens & 115 & 78 & 29 & 33 & & & \\
\hline Avicennia germinans & 108 & 73 & 4 & 7 & & 48 & \\
\hline Landolphia hirsuta & 91 & 73 & 1 & 37 & & & \\
\hline Treculia africana & 58 & 63 & 6 & 1 & & & \\
\hline Cucumis metuliferus & 51 & 61 & 1 & & & & \\
\hline
\end{tabular}




\begin{tabular}{llllllll}
\hline Ficus lutea & 78 & 57 & 6 & 5 & 1 & 26 & \\
Voacanga africana & 100 & 52 & 2 & 59 & & 1 & 7 \\
Psychotria peduncularis & 84 & 48 & 17 & 2 & & & 35 \\
Chrysobalanus ellipticus & 44 & 46 & 1 & 2 & & 4 & \\
Ritchiea capparoides & 51 & 37 & 26 & & & \\
Mammea africana & 26 & 30 & 1 & & & \\
Detarium senegalense & 26 & 29 & 2 & & & \\
Pancovia bijuga & 23 & 23 & 4 & & & 1 \\
Tamarindus indica & 18 & 21 & 1 & & & \multirow{2}{*}{16} \\
Diospyros ferrea & 28 & 65 & 5 & & & \\
Landolphia owariensis & 15 & 65 & 5 & & & \\
Monanthotaxis barteri & 55 & 65 & & & & \\
Drypetes floribunda & 60 & 60 & 1 & 1 & & \\
Aframomum elliotii & 10 & 60 & & & & \\
Uvaria thomasii & 7 & 7 & 1 & & & \\
Synsepalum brevipes & 6 & 7 & & & & \\
Ziziphus mauritiana & 6 & 7 & & & & \\
Zanthoxylum zanthoxyloides & 92 & 6 & 65 & 38 & 1 & 54 \\
Azadirachta indica, neem tree & 63 & 6 & 4 & 6 & 0 & 61 \\
Celtis toka & 5 & 6 & & & & \\
Ficus capensis & 5 & 6 & & & & \\
Annona glauca & 5 & 4 & 2 & & & \\
Calamus deerratus & 10 & 2 & & 10 & & \\
Balanites aegyptiaca & 2 & 2 & & & & \\
Chrysobalanus orbicularis & 2 & 2 & & & & \\
Pseudospondias microcarpa & 2 & 2 & & & & \\
\hline
\end{tabular}

Fo : food; Med : medicinal; Tech : technological; Ag : agronomic; En : energetic; Cul : cultural

Table 3 shows the fidelity index of the 62 species used in the six use categories. Thus, the fidelity index is greater than $50 \%$ for 47 species in the food sector and for only 1 species, namely Calamus deerratus, in the technological field. The rest of the species have fidelity indices below $50 \%$.

The results of the Factorial Correspondence Analysis (FCA) between the use categories and the 62 harvested species are presented in Figure 2. The prior Chi2 independence test showed a statistically significant relationship between the use categories and the 62 species $(\mathrm{X}$-squared $=4975.9, \mathrm{df}=305, p$-value $<2.2 \mathrm{e}-16)$. These results show that the frequency of use of these categories varies according to the species exploited. Table 3 presents the inertia of each of the factorial axes obtained after transformation of the original variables by the FCA. The percentage of inertia of each of the first 2 dimensions (axes) is higher than the average threshold $(1 / 5) * 100=$ $20 \%$. The first two dimensions account for $69 \%$ of the total inertia of the original variables. Thus, all the analyses will be done in the factorial plane composed by these first two dimensions.

Table 3. Distribution of the inertia according to the dimensions (Dim)

\begin{tabular}{llllll}
\hline Axes & Dim1 & Dim 2 & Dim 3 & Dim 4 & Dim 5 \\
\hline Proper value & $\mathbf{0 . 2 7}$ & $\mathbf{0 . 2 1}$ & 0.10 & 0.07 & 0.07 \\
Inertia & $\mathbf{3 9 . 1 2}$ & $\mathbf{2 9 . 8 9}$ & 14.65 & $52.5 \%$ & 5.83 \\
Cumulative percentage of inertia & $\mathbf{3 9 . 1 2}$ & $\mathbf{6 9}$ & 83.66 & 94.16 & 100 \\
\hline
\end{tabular}

Figure 2 of the FCA allows us to isolate three groups (G1, G2, G3). Dimension 1, which accounts for 39.12\%, opposes group 3 in the negative abscissa to groups 1 and 2 in the positive abscissa. Dimension $2(29.89 \%)$ also opposes group 2 in the negative ordinates to group 1 in the positive ordinates.

Group 1 characterizes the species used in the technological and cultural categories. The species Elaeis_guineensis (3.3), Borassus_aethiopum (5.5), Ceiba_pentandra (8.1), which have a contribution above the threshold (average of the absolute contributions $=1.6$ ), represent this group; group 2 includes species for agronomic and energy use characterized by Parkia_biglobosa (7.2), Zanthoxylum_zanthoxyloides (3.9) and group 3 of species for food and medicinal use characterized by Salacia_senegalensis (3.1), Sarcocephalus_latifolius (1.8). This study shows that the species: 
Parinari excelsa, Elaeis guineensis, Borassus aethiopum, Calamus deerratus, Ceiba pentandra, Voacanga africana, Psychotria peduncularis, Phoenix reclinata ( 8 species) are more related to technological and cultural uses;

Azadirachta indica, Zanthoxylum zanthoxyloides, Diospyros ferrea, Mangifera indica, Anacardium occidentale, Parkia biglobosa, Neocarya macrophylla, Dialium guineense, Avicennia germinans, Allophyllus africanus (9 species) are related to agronomic and energy uses;

Atroxima afzeliana, Aframomum cereum, Adansonia digitata, Aframomum elliotii, Annona glauca, Annona senegalensis, Aphania senegalensis, Balanites aegyptiaca, Cola cordifolia, Chrysobalanus ellipticus, Cucumis metuliferus, Chrysobalanus orbicularis, Dryicus topetibicularis, Feticus topetibicularisunda, Deticus topetibicularisea, Feticus topetibicularis, Fevericus senegalensi , Gardenia erubescens, Icacina oliviformis, Landolphia dulcis, Landolphia heudelotii, Landolphia hirsuta, Landolphia owariensis, Mammea africana, Monanthotaxis barteri, Macrosphyra longistyla, Nymphaea -spp, Pancovia bijuga, Passiflora foetida, Pseudospondias microcarpa, Ritchiea capparoides, Synsepalum brevipes, Sorindeia juglandifolia, Sarcocephalus latifolius, Spondias mombin, Saba senegalensis, Salacia senegalensis, Treculia africana, Tamarindus indica, Uvaria chamae, Uvaria thomasii, Vitex doniana, Xylopia aethiopica, Ziziphus mauritiana (45 species) are correlated with food and medicine.

Use categories with an above-average absolute contribution (16.6) have helped build dimensions 1 and 2 . Thus:

$>$ The food use category (31.9) has contributed significantly to building dimension 1;

$>$ The energy use category (24.1) has contributed negatively to dimension 1 .

Dimension 1 opposes food and medicinal categories to energy and agronomic categories and therefore distinguishes the species used in these different categories.

$>$ The cultural use category (37) has contributed positively to building dimension 2 .

Analysis of the results (Figure 2) shows that food and medicinal species in group 3 outnumber the species in groups 1 and 2. These results also show that the nature of the subsistence needs of the Kasa populations is mainly food and medicinal. These results also reveal categories that are highly correlated with each other and with the species. 


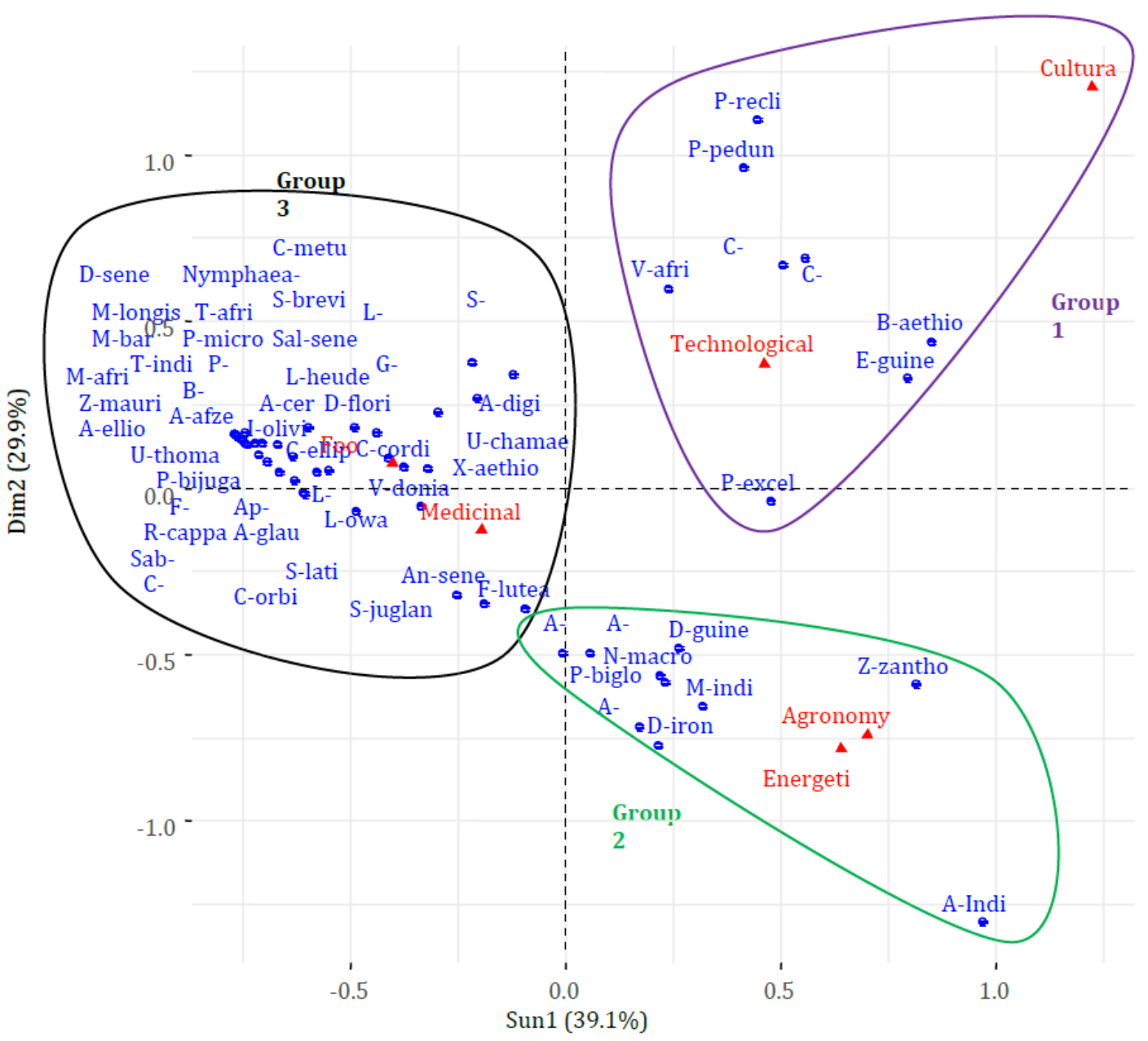

Figure 2. Factoral Correspondence Analysis (FCA) of the matrix of 62 species X 6 use categories

The consensus factors are very high for the six defined use categories. They are between 0.9 and 0.8 (Table 4). This shows that the plant species used and their use modes in the different Diola communities vary very little. Species use is most harmonious in food, cultural, and energy categories, where the consensus factor is 0.98-0.97. The agronomic category, which is strongly marked by female activity, has the lowest consensus factor at 0.89 .

Table 4. Use and their category consensus factor (ICF)

\begin{tabular}{llll}
\hline Use category & Number of citation & Number of Species & ICF \\
\hline Food & 3128 & 62 & 0.98 \\
Energy & 943 & 28 & 0.97 \\
Technological & 716 & 40 & 0.94 \\
medicinal & 678 & 53 & 0.92 \\
Cultural & 325 & 8 & 0.98 \\
Agronomic & 85 & 10 & 0.89 \\
\hline
\end{tabular}

In total, with regard to species use in the context of use value, use categories, organs used, our results show that the local population harvests different organs for multiple uses. Elaeis guineensis, Borassus aethiopum, Mangifera indica, Neocarya macrophylla , Parkia biglobosa, Anacardium occidentale, Ceiba pentandra, Parinari excelsa, are the most used species for the daily needs of the local population.

\section{Discussion}

Our results indicate 62 edible fruit species distributed in 31 families and 54 genera. They present a good generic and specific diversity compared to that of the 75 fruit species belonging to 35 families in the Séguéla region (Ambé, 2001). The results show a dominance of Apocynaceae and Annonaceae, and confirm those obtained by 
(Ambé, 2001) and (Ouattara et al ., 2016).

Among the species studied, Elaeis guineensis (12.24) and Borassus aethiopum (7.56) have the highest use values with a relatively high number of use categories and organs used. Elaeis guineensis has 6 categories and 6 organs, Borassus aethiopum 5 categories and 5 organs. These two species are therefore highly sought after by the populations due to their high socio-economic and cultural values. They are used in the cultural field as well as in profane and sacred rites. Moreover, these species are an essential link in technology (construction of houses, production of means, etc.) and their products are abundantly consumed throughout the sector.

The Anacardiaceae which come in second position, when referring to use value, are essentially represented by Mangifera indica (3.74) with 5 categories and 6 organs and Anacardium occidentale (3.5) with 5 categories and 5 organs. These two species are of great economic importance, in particular Anacardium occidentale, the seeds of which have a high value in national and international markets as well as its alcoholic juice which is highly prized during public ceremonies and events (Djihounouck, 2018). This alcoholic juice is a substitute for palm wine in winter periods when it is scarce.

The results do not show a close proportionality between use categories, organs used and use value. The use of a species depends on its socio-economic importance, availability and accessibility. Therefore, the use value of a family is not proportional to its specific diversity as pointed out by Guèye (2012), Djihounouck et al . (2019). In addition, the species Elaeis guineensis, Borassus aethiopum, Neocarya macrophylla , Parkia biglobosa, Anacardium occidentale, Ceiba pentandra have significant use values between 12.24 and 3.23. The level of exploitation of these species by the local population has been highlighted by Dossou (2010), Lougbegnon et al (2011), Diatta (2016) who have shown that the importance given to a species depends on its capacity to meet the needs of the populations in the different use categories. Borassus aethiopum has a high use value and paradoxically, it is not abundant in our study area. When a species is not abundant and has a high use value, this means that it is under strong pressure as pointed out by Camou-Guerrero et al . (2008), Dossou (2010).

Our results show a certain homogeneity of uses of plant species in the Kasa area with a high consensus factor (ICF) for all use categories. They show six categories of use among the Diola populations in the Kasa area. These are: food, medicinal, technological, agronomic, energetic and cultural.

Food, energy, technological and medicinal categories are the most important in terms of citation with 3128; 943; 716 and 678 respectively. These results are comparable to those obtained by Galeano (2000) among the forest communities in Colombia, where he noted a high citation index for the "food" and "medicinal" categories. These results show that subsistence needs are the primary concern of the populations that exploit plant species. The medicinal category complete the medical centers and districts, which can explain its position.

The agronomic category has the lowest ICF $(0.89)$ as information in this area is mainly held by women, who are not the priority target of the survey. Furthermore, the ICF for the medicinal category is the second lowest (0.92) since only traditional healers and a few initiated people have knowledge of the use of plants.

These results confirm the high value of the ICF for the different use categories of plant species in arid and semi-arid zones of Africa (Gning et al. 2013; Ayantunde et al. 2009; Cheikhyoussef et al. 2011). The ICF provides information on the good knowledge of species exploited by local populations on the one hand, and on natural resources important for the survival and well-being of rural populations on the other hand (Gueye, 2012). The rural population retains a fairly homogeneous knowledge of the uses of plant species transmitted from generation to generation through oral tradition. This knowledge could be a basis for local development policy.

The food category covers the activities of gathering and consumption of plants. All of the species inventoried are food, but their importance varies according to their frequency of citation by informants. Of the 574 citations for the 7 organs used in this category, Elaeis guineensis and Nymphaea spp. are the most frequently cited with 29\% and $27 \%$ respectively, followed by Borassus aethiopum 21\%, Landolphia dulcis $20 \%$ and Adansonia digitata $17 \%$.

The energy studied is that which comes from firewood of woody species, in general, or from charcoal. It covers $42 \%$ of the species inventoried. This high frequency of citation reveals a significant energy demand by the populations and the diversity of species mobilized to meet this need. This category includes the priority activities of rural women, most of whom are housewives. Carrière et al. (2005) showed that among the Betsileo of Madagascar, firewood is the main category of useful plants for domestic use harvested in post-agricultural recruits and ranks first ahead of other uses such as pharmaceuticals, craft and construction.

The technological category concerns the making of means and includes $63 \%$ of species. The species Elaeis guineensis and Borassus aethiopum have the highest citation frequencies with $49 \%$ and $40 \%$ respectively and are 
therefore the most used in the technological field. Their leaves are used for construction (house roof, hut, fence, etc.) and for crafting service products (brooms, baskets, mats, fish traps, basketwork, furniture, fibers, ropes, benches, belts to climb palm trees, ...) ; their trunks or stipes are used to construct bridges, beehives, dikes and habitats (pillar, frame, ceiling, roof, hut). These results confirm those of Sambou et al . (1992) and Blinch (2002). In addition, palm leaves and stipes give these species a high market value (Johnson, 2010).

The medicinal category represents $82 \%$ of the species inventoried. This study has enabled us to identify 96 pathologies among the kasa populations. The species frequently cited by the populations for the treatment of pathologies are: Mangifera indica, Elaeis guineensis, Landolphia dulcis, Parkia biglobosa and Xylopia aethiopica. The frequency of use of a species for disease treatment is an index of reliability of its effectiveness. Some authors even argue that plant species used repeatedly by the population in the medical field are generally effective and interesting in the search for bioactive molecules (Trotter \& Logan, 1986; King et al. , 1996; Guèye, 2012). The fruit species used in the kasa have multiple medicinal uses except Drypetes floribunda, Mammea africana, Atroxima afzeliana , Nymphaea spp , Cucumis metuliferus, Tamarindus indica, Annona glauca, Diospyros ferrea which are only used to treat a single pathology.

The cultural category represents $12 \%$ of the species. It covers sacred or profane rites. The leaves of Elaeis guineensis and Phoenix reclinata are used in dancing initiation ceremonies. Elaeis guineensis provides brooms that certain fetish leaders always hold as a sign of identification at the sacred throne and its traditional wine commonly called "bunuk" is used for worshipping the fetishes traditionally called bəkin in order to enter into communion or set a contract with the ancestors and the gods (Loubelo, 2012). Borassus aethiopum is used as a symbol of prohibition to non-initiated people in a festivity or of recognition to a heritage or in a traditional dance or to create coffins. Psychotria peduncularis is used for blessings. Ceiba pentandra is used to create coffins often with the stem of Carapa Aubl. spp. also reported by Djihounouck (2010).

The agronomic category concerns $15 \%$ of the species. The leaves of Mangifera indica and Ceiba pentandra, and the fruits of Parkia biglobosa are often used as fertilizers for cultivated land. Evaluation of the impact of fertilization by the néré ( Parkia biglobosa) and shea (Vitellaria paradoxa) on sorghum seed yields has shown an increase of $50-70 \%$ in Burkina Faso (Kessler, 1992).

\section{Conclusion}

This study identified the needs of the populations in relation to the 62 fruit species exploited in the Kasa area and highlighted the relationship between these species and the needs of the populations. The various products derived from these species have different uses grouped into six categories. These uses have made it possible to understand the importance of these resources for the local population in all areas of life. The uses of fruit species are relatively homogeneous for the different categories; however, they are more marked for food needs, followed by those of energy, technology, medicine, agronomy and culture. The use values, categories and organs used obtained allowed the identification of the most important species for the populations. In the kasa area, these are Elaeis guineensis, Borassus aethiopum, Mangifera indica, Neocarya macrophylla, Parkia biglobosa, Anacardium occidentale, Ceiba pentandra, Parinari excelsa, Thus, the estimation of the threat to these species by the use value assessed through harvesting has defined these species with the exception of Elaeis guineensis. This species has been little affected by human activities because of its strong predominance in the forest. The study reveals 7 parts of the plants exploited (fruit, wood, leaf, bark, root, flower and exudate) with a preference for fruits with food use. For a sustainable management of these non-cultivate edible and multiple-use fruit species, it is necessary to observe the population dynamics of the exploited species and involve local users in the resource, especially in the search for income.

\section{Acknowledgments}

We are grateful to the village chiefs, guides and above all to the villagers for their full collaboration in the collection of data.

\section{References}

Adam, J. G. (1970). Noms vernaculaires des plantes du Sénégal. Journal d'Agriculture tropicale et de botanique appliquée, 17(7-8-9), 112. https://doi.org/10.3406/jatba.1970.3070

Allabi, A. C., Busiac, K., Ekanmiana, V., \& Bakiono, F. (2011). The use of medicinal plants in self-care in the Agonlin region of Benin. Journal of Ethnopharmacology, 133(1), 234-243. https://doi.org/10.1016/j.jep.2010.09.028

Ambé G. A. (2001). Les fruits sauvages comestibles des savanes guinéennes de Côte d'Ivoire: état de la connaissance par une population locale, les Malinkés. Biotchnol. Agron. Soc. Environ., 5(1), 43-58. 
Ayantunde, A. A., Hiernaux, P., Briejer, M., Udo, H. M. J., \& Tabo, R. (2009). Uses of local plant species by agropastoralists in South-western Niger. Ethnobotany Research \& Applications, 7, 53-66. https://doi.org/10.17348/era.7.0.53-66

Ayessou N.C., Guèye M., Dioh E., Konteye M., Cissé M. \& Dornier M. (2009). Composition nutritive et apport énergétique du fruit de Maerua pseudopetalosa (Gil et Gil-Ben) DeWolf (Capparidaceae), aliment de soudure au Sénégal. Fruits, 64(3), 147-156. https://doi.org/10.1051/fruits/2009010

Ayessou, N. C., Ndiaye, C., Cissé, M., Guèye, M., \& Sakho, M. (2011). Nutritional contribution of some Senegaleseforest fruits running across Soudano-Sahelian zone. Food and Nutrition Sciences, 2(6), 606-612. https://doi.org/10.4236/fns.2011.26085

Berhaut, J. (1967). Flore du Sénégal plus complète avec les forêts humides de la Casamance. Ed. Claire Afrique, Dakar. p. 485.

Berhaut, J. (1979). Flore illustrée du Sénégal. Dicotylédones. Tome 6 Linaceae à Nyphéaceae. Gouvernement du Sénégal-Ministère du développement Rural et de l'Hydraulique, Direction des Eaux et Forêts, Dakar. p. 636.

Bikoué, M. A. C., \& Essomba, H. (2007). Gestion des ressources naturelles fournissant les PFNL alimentaires en Afrique centrale. Produit Forestier Non ligneux. Document de Travail Nㅜ5. Organisation des Nations Unies pour l'Alimentation et l'Agriculture. p. 104.

Blench, R. (2002). Arbres fruitiers sauvages et protégés en préfecture Logone Oriental, S. Tchad. Overseas Development Institute à Londres. p. 23.

Camou-Guerrero, A., Reyes-García, V., Martínez-Ramos, M., \& Casas, A. (2008). Knowledge and use value of plant species in a Rarámuri community: a gender perspective for conservation. Human Ecology, 36(2) 259-272. https://doi.org/10.1007/s10745-007-9152-3

Carriere, S. M., Andrianotahiananahary, H., Ranaivoarivelo, N., \& Randriamalala, J. (2005). Savoir et usages des recrus post-culturales du pays Betsileo: valorisation d'une biodiversité oubliée à Madagascar. VertigO-la revue électronique en sciences de l'environnement, 6(1). https://doi.org/10.4000/vertigo.3047

Cheikhyoussef, A., Ashekele, H., Shapi, M., \& Matengu, K. (2011). Ethnobotanical study of indigenous knowledge on medicinal plant use by traditional healers in Oshikoto region, Namibia. Journal of Ethnobiology and Ethnomedicine, 7-10. https://doi.org/10.1186/1746-4269-7-10

Core, T. R. (2017). R: A language and environment for statistical computing. R Foundation for Statistical Computing, Vienna, Austria. Retrieved from https://www.R-project.org

Cotton, C. M. (1996). Ethnobotany. Principles and Applications. John Wiley \&Sons. p. 424.

Diatta, C. D. (2016), Les plantes utilisées par Les Baïnounk de Djibonker, Région de Ziguinchor (Sénégal) : Diversité et savoirs traditionnels. Thèse doctorat d'Etat, FST, UCAD. p. 127.

Dieng, S. D. (2017). Evaluation des services écosystémiques fournis par Cordyla pinnata (Lepr. Ex A. Rich.) Milne-Redh., Detarium microcarpum Guill. Et Perr. et Detarium senegalense (J.F. Gmel.) : cas de la Forêt Classée de Patako et de ses environs (Centre-Ouest du Sénégal). Thèse de doctorat en Sciences de l'Environnement, Faculté des Sciences et Techniques, Université Cheikh Anta Diop de Dakar. p. 184.

Diop, M. (2011). L'arbre et la Forêt. Usages, préférences, représentations et croyances chez les populations riveraines de la Forêt Classée de Patako (région de Fatick, Sénégal). Thèse Environnement, UCAD. p. 201.

Diop, N., Ndiaye, A., Cisse, M., Dieme, O., Dornier, M., \& Sock, O. (2010). Le ditax (Detarium senegalense J. F. Gmel.): principales caractéristiques et utilisations au Sénégal. Fruits, 65, 293-306. https://doi.org/10.1051/fruits/2010025

Djihounouck, Y. (2010). Ethnobotanique de Carapa Aubl. spp. Chez le Diola du Kasa du Département d'Oussouye (Sénégal). Mémoire de Master, FST, UCAD. p. 38.

Djihounouck, Y., Diop, D., Dieng, S. D., Sané, S., Bassène, C., Mbaye, M. S., \& Noba, K. (2018). Diversité et importance socio-économique des espèces fruitières sauvages Comestibles En Zone Kasa (Sud-Ouest Du Sénégal). European Scientific Journal, ESJ, 14(36), 352-376. https://doi.org/10.19044/esj.2018.v14n36p352

Djihounouck, Y., Diop, D., Bassène, C., Mbaye, M. S., \& Noba, K. (2019). Espèces fruitières spontanées comestibles à usage médicinal dans le Kasa (sénégal). Bulletin de l'IFAN Ch. A. Diop. T. LIV sér. A, 1, $87-112$. 
Dossou, M. E. (2010). Etude floristique, ethnobotanique et proposition d'aménagement de la forêt marécageuse d'Agonvè et zones connexes (Commune de Zagnanado). Mémoire de maitrise en géographie. FLASH/UAC. Ab-Calavi, Bénin. p. 81.

Dossou, M. E., Houessou, G. L., Lougbégnon, O. T., Tenté, A. H. B., \& Codjia, J. T. C. (2012). Étude ethnobotanique des ressources forestières ligneuses de la forêt marécageuse d'Agonvè et terroirs connexes au Bénin, Tropicultura, 30(1), 41-48.

Faucault, M. (1966). Les mots et les choses. Paris, Gallimard. p. 400.

Friedman, J., Yaniv, Z., Dafni, A., \& Palewitch, D. (1986). A preliminary classification of the healing potential of medicinal plants, based on a rational analysis of an ethnopharmacological field survey among Bedouins in the Negev Desert, Israel. Journal of ethnopharmacology, 16(2-3), 275-287. https://doi.org/10.1016/0378-8741(86)90094-2

Galeano, G. (2000). Forest use at the Pacific coast of Chocó, Colombia: A quantitative approach. Economic Botany, 54(3), 358-376. https://doi.org/10.1007/BF02864787

Gning, O. N., Sarr, O., Gueye, M., Akpo, L. E., \& Ndiaye, P. M. (2013). Valeur économique de l'earbre en milieu malinké, Sénégal. Journal of Applied Biosciences, 70, 5617-5631. https://doi.org/10.4314/jab.v70i1.98765

Grivetti, L. E., Frentzel, C. J., Ginsberg, K. E., Howell, K. L., \& Ogle, B. M. (1987). Bush foods and edible weeds of agriculture: perspectives of dietary use of wild plants in Africa, their role in maintaining human nutritional status and implication for agricultural development, In R. Akhtar (Ed.), Health and diseases in Tropical Africa (51-81). London: Harwood.

Guèye, M. (2012). Contribution à l'étude ethnobotanique chez les Malinké de la communauté rurale de Tomboronkoto (région de Kédougou) et valorisation des collections historiques de l'Herbier de l'Institut fondamental d'Afrique noire Cheikh Anta Diop (IFAN Ch. A. Diop) / UCAD. Thèse doctorat d'Etat, FST, $U C A D, 18,142$.

Jiofack, T., Ayissi, I., Fokunang, C., Guedje, N., \& Kemeuze, V. (2009). Ethnobotany and phytomedicine of the upper Nyong Valley forest in Cameroon. African Journal of Pharmacy and pharmacology, 3(4), 144-150.

Jiofack, T., Fokunang, C., Guedje, N., Kemeuze, V., Fongnzossie, E., Nkongmeneck, B. A., Mapongmetsem, P. M., \& Tsabang, N. (2010). Ethnobotanical uses of medicinal plants of two ethnoecological regions Cameroon. International Journal of Medicine and Medical Sciences, 2(3), 60-79. http://www.academicjournals.org/ijmms

Kerharo, J., \& Adam, J. G. (1974). La pharmacopée Sénégalaise Traditionnelle: plantes médicinales et toxiques. Ed. Vigot Frères, Paris. p. 1011.

Kessler, J. J. (1992). The influence of karité (Vitellaria paradoxa) and néré (Parkia globosa) trees on sorghum production in Burkina Faso. Agroforestry Systems, 17, 97-118. https://doi.org/10.1007/BF00053116

King, S. R., Carlson, T. J., \& Moran, K. (1996). Biological diversity, indigenous knowledge, drug discovery and intellectual property rights: creating relationships, Journal of Ethnopharmacology, 51, 45-57. https://doi.org/10.1016/0378-8741(95)01349-0

Lebrun, J. P., \& Stork, A. L. (1991, 1992, 1995, 1997). Enumération des plantes à fleurs d'Afrique tropicale, Genève: Conservatoire et Jardin botanique de Genève. p. 1-4.

Loubelo, E. (2012). Impact des produits forestiers non ligneux (PFNL) sur l'économie des ménages et la sécurité alimentaire : cas de la République du Congo. Thèse de doctorat, Economies et Finances. Université Rennes 2. Français. NNT : 2012REN20008. AE 2241. p. 231.

Lougbegnon, T. O., Tente, B. A. H., Amontcha, M., \& Codjia, J. T. C. (2011). Importance culturelle et valeur d'usage des ressources végétales de la réserve forestière marécageuse de la vallée de Sitatunga et zones connexes. Bulletin de la Recherche Agronomique du Bénin, 70, 35-46.

Malaisse, F. (1992). La gestion des produits sauvages comestibles. Défis- Sud, 7, 18-19.

Martin, G. J. (1995). Ethnobotany. A method manual. London: Chapman \& Hall. p. 268. https://doi.org/10.1007/978-1-4615-2496-0

Negura, L. (2006). L'analyse de contenu dans l'étude des représentations sociales. Sociologies. https://doi.org/10.4000/sociologies.993

Ouattara, N. D., Gaille, E., Stauffer, F. W., \& Bakayoko, A. (2016). Diversité floristique et ethnobotanique des 
plantes sauvages comestibles dans le Département de Bondoukou (Nord- Est de la Côte d'Ivoire). Journal of Applied Biosciences, 98, 9284-9300. https://doi.org/10.4314/jab.v98i1.5

Philips, O., \& Gentry, A. H. (1993). The useful plants of Tambopata, Peru. II Statistical hypothesis tests with a new quantitative technique. Economic Botany, 47(1), 33-43. https://doi.org/10.1007/BF02862204

Sambou, B., Lawesson, J. E., \& Barfod, A. S. (1992). Borassus aethiopum, a threatened multiple purpose palm in Senegal. Principes, 36(3), 148-155.

Sofowora, A. (1982). Medicinal plants and traditional Medicine in West Africa. John Wiley and Sons, New York. p. 256.

Trotter, R. T., \& Logan, M. H. (1986). Informant Consensus: a New Approach for Identifying Potentially Effective Médicinal Plants. Bedfore Hills: New York. p. 91-112. https://doi.org/10.4324/9781315060385-6

Van den Eynden, V., Van Damme, P., \& De Wolf, J. (1994). Inventaire et modelage de la gestion du couvert végétal pérenne dans une zone forestière du sud du Sénégal. Rapport final. Partie $\mathrm{C}$ Etude ethnobotanique. Université de Gand, Belgique. p. 33-99.

Van Der Berghen, C. (1988). Flore illustrée du Sénégal. Monocotylédones et Ptéridophytes. Tome 10. Gouvernement du Sénégal-Ministère du développement Rural et de l'Hydraulique, Direction des Eaux et Forêts, Dakar. p. 523.

Annex: List of plant species used (abbreviated) for AFC

\begin{tabular}{|c|c|c|c|c|c|}
\hline $\begin{array}{l}\text { Allophyllus } \\
\text { africanus (A-afri) }\end{array}$ & $\begin{array}{l}\text { Drypetes } \\
\text { floribunda (D-flori) }\end{array}$ & $\begin{array}{l}\text { Passiflora } \\
\text { foetida (P-foeti) }\end{array}$ & $\begin{array}{l}\text { Allophyllus } \\
\text { africanus } \\
\text { (A-afri) }\end{array}$ & $\begin{array}{l}\text { Drypetes } \\
\text { floribunda } \\
\text { (D-flori) }\end{array}$ & $\begin{array}{l}\text { Passiflora } \\
\text { foetida (P-feti) }\end{array}$ \\
\hline $\begin{array}{l}\text { Atroxima afzeliana } \\
\text { (A-afze) }\end{array}$ & $\begin{array}{l}\text { Dialium } \\
\text { guineense (D-guine) }\end{array}$ & $\begin{array}{l}\text { Pseudospondias } \\
\text { microcarpa (P-micro) }\end{array}$ & $\begin{array}{l}\text { Atroxima } \\
\text { afzeliana } \\
\text { (A-afze) }\end{array}$ & $\begin{array}{l}\text { Dialium } \\
\text { guineense } \\
\text { (D-guine) }\end{array}$ & $\begin{array}{l}\text { Pseudospondias } \\
\text { microcarpa } \\
\text { (P-micro) }\end{array}$ \\
\hline $\begin{array}{l}\text { Aframomum } \\
\text { cereum (A-cer) }\end{array}$ & $\begin{array}{l}\text { Detarium } \\
\text { senegalense (D-sene) }\end{array}$ & $\begin{array}{l}\text { Psychotria } \\
\text { peduncularis (P-pedun) }\end{array}$ & $\begin{array}{l}\text { Aframomum } \\
\text { cereum (A-cer) }\end{array}$ & $\begin{array}{l}\text { Detarium } \\
\text { senegalense } \\
\text { (D-sene) }\end{array}$ & $\begin{array}{l}\text { Psychotria } \\
\text { peduncularis } \\
\text { (P-pedun) }\end{array}$ \\
\hline $\begin{array}{l}\text { Adansonia } \\
\text { digitata (A-digi) }\end{array}$ & $\begin{array}{l}\text { Elaeis } \\
\text { guineensis (E-guine) }\end{array}$ & $\begin{array}{l}\text { Phoenix } \\
\text { reclinata (P-recli) }\end{array}$ & $\begin{array}{l}\text { Adansonia } \\
\text { digitata } \\
\text { (A-digi) }\end{array}$ & $\begin{array}{l}\text { Elaeis } \\
\text { guineensis } \\
\text { (E-guine) }\end{array}$ & $\begin{array}{l}\text { Phoenix } \\
\text { reclinata } \\
\text { (P-recli) }\end{array}$ \\
\hline $\begin{array}{l}\text { Aframomum } \\
\text { elliotii (A-ellio) }\end{array}$ & Ficus capensis (F-cap) & $\begin{array}{l}\text { Ritchiea capparoides } \\
\text { (R-cappa) }\end{array}$ & $\begin{array}{l}\text { Aframomиm } \\
\text { elliotii (A-ellio) }\end{array}$ & $\begin{array}{l}\text { Ficus capensis } \\
\text { (F-cap) }\end{array}$ & $\begin{array}{l}\text { Ritchiea } \\
\text { capparoides } \\
\text { (R-cappa) }\end{array}$ \\
\hline $\begin{array}{l}\text { Avicennia } \\
\text { germinans (A-ger) }\end{array}$ & Ficus lutea (F-lutea) & $\begin{array}{l}\text { Synsepalum } \\
\text { brevipes (S-brevi) }\end{array}$ & $\begin{array}{l}\text { Avicennia } \\
\text { germinans } \\
\text { (A-ger) }\end{array}$ & $\begin{array}{l}\text { Ficus lutea } \\
\text { (F-lutea) }\end{array}$ & $\begin{array}{l}\text { Synsepalum } \\
\text { brevipes } \\
\text { (S-brevi) }\end{array}$ \\
\hline $\begin{array}{l}\text { Annona } \\
\text { glauca (A-glau) }\end{array}$ & $\begin{array}{l}\text { Gardenia } \\
\text { erubescens (G-eru) }\end{array}$ & $\begin{array}{l}\text { Sorindeia } \\
\text { juglandifolia (S-juglan) }\end{array}$ & $\begin{array}{l}\text { Annona glauca } \\
\text { (A-glau) }\end{array}$ & $\begin{array}{l}\text { Gardenia } \\
\text { erubescens } \\
\text { (G-eru) }\end{array}$ & $\begin{array}{l}\text { Sorindeia } \\
\text { juglandifolia } \\
\text { (S-juglan) }\end{array}$ \\
\hline $\begin{array}{l}\text { Azadirachta } \\
\text { indica (A-Indi) }\end{array}$ & $\begin{array}{l}\text { Icacina } \quad \text { oliviformis } \\
\text { (I-olivi) }\end{array}$ & $\begin{array}{l}\text { Sarcocephalus latifolius } \\
\text { (S-lati) }\end{array}$ & $\begin{array}{l}\text { Azadirachta } \\
\text { indica (A-Indi) }\end{array}$ & $\begin{array}{l}\text { Icacina } \\
\text { oliviformis } \\
\text { (I-olivi) }\end{array}$ & $\begin{array}{l}\text { Sarcocephalus } \\
\text { latifolius (S-lati) }\end{array}$ \\
\hline $\begin{array}{l}\text { Anacardium } \\
\text { occidentale (A-occi) }\end{array}$ & $\begin{array}{l}\text { Landolphia } \\
\text { dulcis (L-dul) }\end{array}$ & $\begin{array}{l}\text { Spondias } \\
\text { mombin (S-mom) }\end{array}$ & $\begin{array}{l}\text { Western } \\
\text { anacardium } \\
\text { (A-occi) }\end{array}$ & $\begin{array}{l}\text { Landolphia } \\
\text { dulcis (L-dul) }\end{array}$ & $\begin{array}{l}\text { Mombin } \\
\text { spondias } \\
\text { (S-mom) }\end{array}$ \\
\hline $\begin{array}{l}\text { Annona } \\
\text { senegalensis (An-sene) }\end{array}$ & $\begin{array}{l}\text { Landolphia } \\
\text { heudelotii (L-heude) }\end{array}$ & $\begin{array}{l}\text { Saba } \\
\text { senegalensis (Sab-sene) }\end{array}$ & $\begin{array}{l}\text { Annona } \\
\text { senegalensis } \\
\text { (An-sene) }\end{array}$ & $\begin{array}{l}\text { Landolphia } \\
\text { heudelotii } \\
\text { (L-heude) }\end{array}$ & $\begin{array}{l}\text { Saba } \\
\text { senegalensis } \\
\text { (Sab-sene) }\end{array}$ \\
\hline $\begin{array}{l}\text { Aphania senegalensis } \\
\text { (Ap-sene) }\end{array}$ & $\begin{array}{l}\text { Landolphia } \\
\text { hirsuta (L-hirsu) }\end{array}$ & $\begin{array}{l}\text { Salacia } \\
\text { senegalensis (Sal-sene) }\end{array}$ & $\begin{array}{l}\text { Aphania } \\
\text { senegalensis } \\
\text { (Ap-sene) }\end{array}$ & $\begin{array}{l}\text { Landolphia } \\
\text { hirsuta } \\
\text { (L-hirsu) }\end{array}$ & $\begin{array}{l}\text { Salacia } \\
\text { senegalensis } \\
\text { (Sal-sene) }\end{array}$ \\
\hline $\begin{array}{l}\text { Balanites } \\
\text { aegyptiaca (B-aegyp) }\end{array}$ & $\begin{array}{l}\text { Landolphia owariensis } \\
\text { (L-owa) }\end{array}$ & $\begin{array}{l}\text { Treculia } \\
\text { (T-afri) }\end{array}$ & $\begin{array}{l}\text { Balanites } \\
\text { aegyptiaca }\end{array}$ & $\begin{array}{l}\text { Landolphia } \\
\text { owariensis }\end{array}$ & $\begin{array}{l}\text { Treculia africana } \\
\text { (T-afri) }\end{array}$ \\
\hline
\end{tabular}




\begin{tabular}{|c|c|c|c|c|c|}
\hline & & & (B-aegyp) & (L-owa) & \\
\hline $\begin{array}{l}\text { Borassus } \\
\text { aethiopum (B-aethio) }\end{array}$ & $\begin{array}{l}\text { Mammea } \\
\text { africana (M-afri) }\end{array}$ & $\begin{array}{l}\text { Tamarindus } \\
\text { indica } \text { (T-indi) }\end{array}$ & $\begin{array}{l}\text { Borassus } \\
\text { aethiopum } \\
\text { (B-aethio) }\end{array}$ & $\begin{array}{l}\text { Mammea } \\
\text { africana } \\
\text { (M-afri) }\end{array}$ & $\begin{array}{l}\text { Tamarindus } \\
\text { indica (T-indi) }\end{array}$ \\
\hline $\begin{array}{ll}\text { Cola } & \text { cordifolia } \\
\text { (C-cordi) }\end{array}$ & $\begin{array}{l}\text { Monanthotaxis } \\
\text { barteri (M-bar) }\end{array}$ & $\begin{array}{l}\text { Uvaria } \\
\text { chamae (U-chamae) }\end{array}$ & $\begin{array}{l}\text { Cola cordifolia } \\
\text { (C-cordi) }\end{array}$ & $\begin{array}{l}\text { Monanthotaxis } \\
\text { barteri (M-bar) }\end{array}$ & $\begin{array}{l}\text { Uvaria chamae } \\
\text { (U-chamae) }\end{array}$ \\
\hline $\begin{array}{l}\text { Calamus } \\
\text { deerratus (C-deer) }\end{array}$ & $\begin{array}{l}\text { Mangifera } \\
\text { indica (M-indi) }\end{array}$ & $\begin{array}{l}\text { Uvaria } \\
\text { thomasii (U-thoma) }\end{array}$ & $\begin{array}{l}\text { Calamus } \\
\text { deerratus } \\
\text { (C-deer) }\end{array}$ & $\begin{array}{l}\text { Mangifera } \\
\text { indica (M-indi) }\end{array}$ & $\begin{array}{l}\text { Uvaria thomasii } \\
\text { (U-thoma) }\end{array}$ \\
\hline $\begin{array}{l}\text { Chrysobalanus } \\
\text { ellipticus (C-ellip) }\end{array}$ & $\begin{array}{l}\text { Macrosphyra } \\
\text { longistyla (M-longis) }\end{array}$ & $\begin{array}{l}\text { Voacanga africana } \\
\text { (V-afri) }\end{array}$ & $\begin{array}{l}\text { Chrysobalanus } \\
\text { ellipticus } \\
\text { (C-ellip) }\end{array}$ & $\begin{array}{l}\text { Macrosphyra } \\
\text { longistyla } \\
\text { (M-longis) }\end{array}$ & $\begin{array}{l}\text { Voacanga } \\
\text { africana (V-afri) }\end{array}$ \\
\hline $\begin{array}{l}\text { Cucumis } \\
\text { metuliferus (C-metu) }\end{array}$ & $\begin{array}{l}\text { Neocarya } \\
\text { macrophylla (N-macro) }\end{array}$ & Vitex doniana (V-donia) & $\begin{array}{l}\text { Cucumis } \\
\text { metuliferus } \\
\text { (C-metu) }\end{array}$ & $\begin{array}{l}\text { Neocarya } \\
\text { macrophylla } \\
\text { (N-macro) }\end{array}$ & $\begin{array}{l}\text { Vitex doniana } \\
\text { (V-donia) }\end{array}$ \\
\hline $\begin{array}{l}\text { Chrysobalanus } \\
\text { orbicularis (C-orbi) }\end{array}$ & Nymphaea-spp & $\begin{array}{l}\text { Xylopia } \\
\text { aethiopica (X-aethio) }\end{array}$ & $\begin{array}{l}\text { Chrysobalanus } \\
\text { orbicularis } \\
\text { (C-orbi) }\end{array}$ & Nymphaea -spp & $\begin{array}{l}\text { Xylopia } \\
\text { aethiopica } \\
\text { (X-aethio) }\end{array}$ \\
\hline $\begin{array}{l}\text { Ceiba } \\
\text { pentandra (C-pentan) }\end{array}$ & $\begin{array}{ll}\text { Parkia } & \text { biglobosa } \\
\text { (P-biglo) } & \end{array}$ & $\begin{array}{l}\text { Ziziphus mauritiana } \\
\text { (Z-mauri) }\end{array}$ & $\begin{array}{l}\text { Ceiba } \\
\text { pentandra } \\
\text { (C-pentan) }\end{array}$ & $\begin{array}{l}\text { Parkia } \\
\text { biglobosa } \\
\text { (P-biglo) }\end{array}$ & $\begin{array}{l}\text { Ziziphus } \\
\text { mauritiana } \\
\text { (Z-mauri) }\end{array}$ \\
\hline Celtis toka (C-toka) & $\begin{array}{l}\text { Pancovia } \\
\text { bijuga (P-bijuga) }\end{array}$ & $\begin{array}{l}\text { Zanthoxylum } \\
\text { zanthoxyloides } \\
\text { (Z-zantho) }\end{array}$ & $\begin{array}{l}\text { Celtis toka } \\
\text { (C-toka) }\end{array}$ & $\begin{array}{l}\text { Pancovia } \\
\text { bijuga } \\
\text { (P-bijuga) }\end{array}$ & $\begin{array}{l}\text { Zanthoxylum } \\
\text { zanthoxyloides } \\
\text { (Z-zantho) }\end{array}$ \\
\hline $\begin{array}{l}\text { Diospyros } \\
\text { ferrea (D-fer) }\end{array}$ & $\begin{array}{l}\text { Parinari } \\
\text { excelsa }(\mathrm{P}-\mathrm{excel})\end{array}$ & & $\begin{array}{l}\text { Diospyros } \\
\text { ferrea (D-fer) }\end{array}$ & $\begin{array}{l}\text { Parinari } \\
\text { excelsa } \\
\text { (P-excel) }\end{array}$ & \\
\hline
\end{tabular}

\section{Copyrights}

Copyright for this article is retained by the author(s), with first publication rights granted to the journal.

This is an open-access article distributed under the terms and conditions of the Creative Commons Attribution license (http://creativecommons.org/licenses/by/4.0/). 